\title{
INTERNACIONALIZACIÓN Y REFORMAS DEL SISTEMA DE EDUCACIÓN SUPERIOR EN CHINA
}

\author{
Manuel Pérez García ${ }^{1}$
}

Fecha de recepción: 30 de marzo de 2016. Fecha de aceptación: 27 de junio de 2016.

\begin{abstract}
RESUMEN
El objetivo de este trabajo es presentar una visión general de los aspectos que articulan la relación estratégica existente entre Europa y China, centrándose en la cooperación y movilidad científica promovida por la Comisión Europea y el Ministerio de Educación de China, durante los últimos años. La visión comparada entre China y los Estados Unidos de América en lo referente a movilidad e intercambios científicos resulta necesaria, ya que el flujo migratorio de origen chino ha tenido siempre como destino principal suelo estadounidense. El objetivo de la cooperación sino-europea se basa en la introducción de reformas en el sistema de educación superior con el fin de erradicar la "fuga de cerebros" que ha afectado dramáticamente a la nación asiática y a los países miembros de la Unión Europea.
\end{abstract}

Palabras clave: educación superior, reforma educativa, fuga de cerebros, movilidad internacional, intercambio científico.

Clasificación JEL: I21, I28.

\section{INTERNATIONALIZATION AND REFORMS in the Higher Education System in China}

\begin{abstract}
The objective of this paper is to provide a general overview of the aspects that define the strategic relationship between Europe and China, centered on the scientific cooperation and mobility promoted by the European Commission and the Chinese Ministry of Education in recent years. The shared vision of China and the United States in terms of scientific mobility and exchange programs is necessary, as migration flows of Chinese origin have always been primarily destined for United States soil. The objective of SinoEuropean cooperation is based on reforming the higher education system to do away with the "brain drain" that has had a dramatic impact on the Asian nation and member countries of the European Union.
\end{abstract}

Key Words: Higher education, education reform, brain drain, international mobility, scientific exchange programs.

1 Universidad Renmin de China. Correo electrónico: manuel.perez@eui.eu 


\section{INTERNATIONALISATION ET RÉFORMES DU SYSTEME D'ÉDUCATION SUPÉ- RIEURE EN CHINE}

\section{Résumé}

L'objectif de ce travail est de présenter une vue générale des aspects qui articulent la relation stratégique entre l'Europe et la Chine en se centrant sur la coopération et la mobilité scientifique promue par la Commission européenne et le Ministère de l'Éducation chinois au cours des dernières années. La vue comparée de la Chine et des États-Unis d'Amérique en ce qui concerne la mobilité et les échanges scientifiques s'avère nécessaire, étant donné que le flux migratoire d'origine chinoise a toujours eu pour destination principale le sol étasunien. La coopération sino-européenne se base sur l'introduction de réformes dans le système d'éducation supérieure dans le but d'éradiquer la "fuite de matière grise " qui a affecté dramatiquement la nation asiatique et les pays membres de l'Union européenne.

Mots clés: éducation supérieure, réforme éducative, fuite de matière grise, mobilité internationale, échange scientifique.

\section{INTERNACIONALIZAÇÃO E REFORMAS DO SISTEMA DE EDUCAÇÁO SUPERIOR NA CHINA}

\section{Resumo}

O objetivo deste trabalho é apresentar uma visão geral dos aspectos que articulam a relação estratégica existente entre a Europa e a China, concentrando-se na cooperação e mobilidade científica promovida pela Comissão Europeia e o Ministério de Educação da China durante os últimos anos. É necessária a visão comparada entre China e Estados Unidos da América no que diz respeito à mobilidade e aos intercâmbios científicos, já que o fluxo migratório de origem chinês teve sempre como principal destino o solo estadunidense. $\mathrm{O}$ objetivo da cooperação sino-europeia se baseia na introdução de reformas no sistema de educação superior com o propósito de erradicar a "fuga de cérebros" que afetou dramaticamente a nação asiática e aos países membros da União Europeia.

Palavras-chave: educação superior, reforma educativa, fuga de cérebros, mobilidade internacional, intercâmbio científico.

\section{中国高等教育体系的国际化及改革}

摘要

本文将着眼于欧盟委员会和中国教育部近年来共同推动的合作及交流阐 释中欧战略关系中的各个方面。美国一直都是中国人移民的首选之地, 因 此, 中美间学生交换及科学交流的对比研究是十分有必要的。中欧合作的 目标基于高等教育体系的改革以防止人才流失。此前中国和欧盟国家饱受 人才流失的困扰。

关键词：高等教育，教育改革，人才流失，国际交换，科学交流 


\section{INTRODUCCIÓN: MOVILIDAD CIENTÍFICA Y COOPERACIÓN ENTRE EUROPA Y CHINA}

Ante todo es preciso mencionar que el objetivo principal de este trabajo es el de presentar una visión general sobre la progresiva internacionalización del sistema de educación superior de China y la implantación de estudios globales e internacionales. El establecimiento de tales estudios en China tiene su origen en los últimos años y su reciente creación está vinculada a las actuales reformas relativas a la internacionalización de departamentos, escuelas, facultades e institutos de investigación.

Pese a que se presentarán algunos ejemplos y estadísticas generales, inéditas y de elaboración propia, de expertos extranjeros en instituciones chinas y algunas comparaciones de programas de movilidad científica en Europa y China, el núcleo de este artículo está orientado hacia un análisis teórico más que empírico.

Por lo anterior, la pregunta fundamental es jverdaderamente se está produciendo en China una reforma a gran escala en lo que respecta a la internacionalización de su sistema de educación superior? De esta manera, se presentarán los orígenes de la internacionalización de las estructuras académicas de China remontándonos al momento en que sinólogos estadounidenses abrieron nuevos estudios de área sobre el país. Este análisis podría ayudar a comprender mejor el nuevo contexto de las Relaciones Internacionales, a través de las cuales, a partir de 1989 el sistema internacional se redefinió como un mundo multipolar caracterizado por estructuras que se superponen a nivel económico, político y social en las que sobresalen los conceptos de diversidad y movilidad (Geeraerts, 2011: 57-67).

Con el fin de dar más detalles sobre el significado del concepto de multipolaridad, en el que se basan las actuales relaciones entre China y Europa, es importante mencionar el nuevo papel de China desde su impresionante crecimiento económico en las últimas décadas y su peso en la gobernanza global (Shen, 2009: 33-55). Beijing, como una de las principales capitales mundiales,

El presente artículo forma parte del proyecto Global Encounters between China and Europe: Trade Networks, Consumption and Cultural Exchanges in Macau and Marseille (1680-1840), dirigido por Manuel Pérez García y financiado por el ERC (European Research Council)-Starting Grant, GECEM Project - 679371 .

Igualmente coopera en la presente investigación el proyecto del Ministerio de Economía y Competitividad de España: Globalización ibérica: redes entre Asia y Europa y los cambios en las pautas de consumo en Latinoamérica (HAR2014- 53797-P, director: Bartolomé Yun Casalilla). Manuel Pérez García forma parte de este proyecto como investigador del equipo. 
se ha vuelto más activa al establecer relaciones multilaterales de cooperación con el resto del mundo. La capital china se ha convertido en sede principal del nuevo sistema político que bien se puede definir como global.

Dentro de este nuevo marco ya no hay un centro de gravedad en términos políticos y económicos, sino un sistema mundial policéntrico en el que China y Europa, entre otros territorios, conforman una nueva estructura en la cual las viejas rencillas heredadas de la Guerra Fría han quedado obsoletas. Europa ha adoptado una política más pragmática hacia China en relación con el nuevo orden internacional debido a la diferente idiosincrasia de China en términos culturales y sociales, y la reciente migración de comunidades de origen chino a Europa. Estos son los principales temas que han comenzado a ser abordados con respecto a la asociación estratégica entre Europa y China. En este sentido, es importante presentar las nuevas políticas emprendidas por la Unión Europea (UE) y China en los últimos diez años en que ambos poderes políticos se reconocieron mutuamente como socios estratégicos.

Dicho reconocimiento cristalizó en términos de cooperación académica y científica con la apertura del Euraxess-Links en Beijing en 2008. Desde esa fecha hasta hoy la movilidad académica y científica de Europa a China ha aumentado de manera exponencial. El Euraxess-Links actúa como una plataforma académica para proporcionar información sobre las políticas europeas de investigación, oportunidades de financiación para investigaciones, cooperación internacional y movilidad para investigadores establecidos en países de regiones donde Euraxess tiene sus oficinas: Europa, América Latina y Asia.

\section{CAMINO HACIA LA "INTERNACIONALIZACIÓN": INSTITUCIONALIZACIÓN DE ESTUDIOS GLOBALES EN CHINA}

Antes de los años noventa todos los estudios sobre China, especialmente los realizados por eruditos occidentales, en concreto los de especialistas con sede en universidades e instituciones de investigación de los Estados Unidos de América, estaban fuertemente marcados por el contexto político de la Guerra Fría y el statu quo de las relaciones sino-estadounidenses (Shambaugh, 1993). Fue entonces cuando en la comunidad de académicos estadounidenses se despertó un gran interés hacia los estudios sobre China.

Destacados especialistas como H.B. Morse (1910-1918), J.K. Fairbank (1950) y Orville Schell (1977) durante los siglos XIX y Xx comenzaron sus estudios de lengua, cultura, historia, economía y política de la nación asiática. Fairbank fue alumno de Morse y también estudió en la Universidad de 
Tsinghua, bajo la dirección del destacado historiador Tsiang Tingfu quien le introdujo en el mundo académico chino. Éstos, por supuesto, fueron figuras notables en los estudios sobre China, los cuales abrieron un nuevo camino a posteriores generaciones que inspiraron a un nuevo y entusiasta grupo de sinólogos en los Estados Unidos de América.

En consecuencia, cuando en el presente texto se mencione estudios chinos, se estará aludiendo al carácter interdisciplinar del propio término. Sin embargo, no debe dejarse de lado que tanto estudios sobre China en Occidente, así como estudios occidentales y/o globales en China, han estado marcados por el mundo ideológicamente polarizado del siglo xx. Personajes como John Lindbeck han analizado la situación de los estudios contemporáneos en los Estados Unidos de América y retratado las contribuciones académicas y la comprensión de estudios sobre China, los cuales sufrieron un fuerte impacto durante la Guerra Fría y la época posterior a la fundación de la República Popular de China (RPCh) en 1949 (Lindbeck, 1971).

El nuevo periodo de relaciones sino-estadounidenses a principios de los años setenta, durante el gobierno de Richard Nixon, seguido de la administración de James Carter, y las nuevas reformas de Deng Xiaoping, marcó la apertura en China y, por tanto, una nueva generación de sinólogos. En consecuencia, un notable grupo de académicos estadounidenses, de origen no chino, desarrolló una buena competencia en el dominio del chino-mandarín, pudiendo publicar en revistas especializadas chinas estando al mismo tiempo familiarizados con el sistema de educación superior chino. Obviamente en el último siglo los eruditos estadounidenses constituyeron el primer grupo que mostró un gran interés por estudiar la lengua y cultura china e incluso desplazarse al país en cuestión.

La movilidad tanto de investigadores como sinólogos estadounidenses para llevar a cabo su investigación en China y aprender el idioma y cultura tiene su origen en el curso de los hechos y acontecimientos históricos que China y Estados Unidos de América, principalmente en el área de California, compartieron desde mediados del siglo xIx e inicios del siglo xx. Por ejemplo, las disposiciones realizadas dentro de la firma del Tratado de Wanghia (1844), después de la Primera Guerra del Opio, entre China y los Estados Unidos de América en relación con los juicios civiles, en especial lo relativo al pago de deudas correspondientes al comercio entre ambas naciones (Morse, 1921; Remer, 1928). Igualmente, otro buen ejemplo para observar cómo las relaciones entre ambas naciones comenzaron a consolidarse históricamente es el relativo a la llegada de los primeros inmigrantes (Hu-Dehart, 1998) de China a los Estados Unidos de América. No es sorprendente que la primera comunidad 
internacional de académicos en abrir centros de investigación e implementar nuevos planes de estudios sobre Asia y China fuese la estadounidense.

Sin embargo, debido a los hechos históricos que acaecieron a lo largo del siglo xx desde la caída de la dinastía Qing, la fundación de la RPch y las posteriores limitaciones para desarrollar vínculos con Occidente, hizo que el número de académicos que llegaron a China fuese marginal. Progresivamente a finales de los ańos setenta y principios de los ochenta, con la "Política de Puertas Abiertas" avalada por Deng Xiaoping, se logró que el número de estudiosos estadounidenses en ir a China aumentase nuevamente. La mayoría de investigadores que pudieron hacerlo eran apoyados por un patrocinador estadounidense, dentro de un programa de becas que cubría gastos de manutención e investigación. El número de investigadores que llegaron sin ese apoyo económico para residir en una universidad china como estudiantes de master, doctorado, becario postdoctoral, conferenciante, profesor asistente, profesor asociado o profesor, trabajando bajo el amparo de un contrato local chino, era prácticamente nulo.

Ahora, la pregunta a responder sería: ¿̧ha cambiado mucho desde aquellos días el número de profesores extranjeros que actualmente trabajan como personal docente e investigador residiendo permanentemente en China? Como se podrá apreciar más adelante sólo ha habido un incremento mínimo en el número de expertos extranjeros ${ }^{3}$ que trabajan actualmente en universidades chinas. Anteriormente no había esta posibilidad, pudiendo sólo los foreign experts colaborar en universidades y centros de investigación como profesores o investigadores visitantes a través de estancias cortas. Entonces, ¿¿cuál debe ser el esfuerzo que las universidades chinas e instituciones académicas deben hacer para atraer a expertos extranjeros con el fin de impulsar y fomentar un entorno e imagen internacional de las propias universidades chinas dentro del contexto global actual?

Los estudios sobre la China contemporánea, especialmente los relativos a su historia en el siglo xx, en los Estados Unidos de América, y por extensión en Europa, han abarcado principalmente el periodo de la Guerra Fría hasta principios de los años noventa. Tales investigaciones están, de este modo, fuertemente influenciadas por el contexto político. Ha habido algunos errores de percepción o "ilusiones" (Mackerras, 2000; Said, 1978) construidas y fomentadas a través de medios de comunicación y líderes políticos ameri-

3 Aquí el gobierno chino emplea el término foreign experts para referirse al personal docente e investigador extranjero que, a partir del año 2012 tras la reforma del Ministerio de Educación, se asentó en universidades chinas. No se refiere, por tanto, al grupo de expatriados que se instalan en cuerpos diplomáticos y empresas multinacionales. 
canos mediante la inserción en la opinión pública de la idea de que estudiosos, que desarrollaron estudios de China en los Estados Unidos de América, pertenecían a movimientos transgresores fuera de lo políticamente correcto o una conspiración comunista contra el bloque occidental. Este tema muestra como, en ciertas ocasiones, los estudios chinos han estado bajo el pensamiento especulativo y excesivo sesgo político. Harry Harding (1993: 14-42) reclama más colaboración en investigación y cooperación entre disciplinas científicas, entre países y círculos que desarrollan y planifican políticas públicas, especialmente a través de los think-tanks.

No obstante, todavía existe un gran vacío en investigaciones que fomenten dicha participación interdisciplinaria. Sin duda hoy día la realización de investigaciones en China y sobre China es más fácil que la desarrollada durante el siglo xx, debido a la actual calma política y la disminución de tensiones entre China y países occidentales. El efecto positivo de esta relativa calma se manifiesta en la llegada al país asiático de expertos procedentes de países occidentales. Sin embargo, el debate se ha desplazado hacia cuestiones relacionadas con las condiciones de trabajo, en especial las referentes a logística, infraestructura y financiación de investigación para apoyar la labor realizada por expertos extranjeros que han llegado en los últimos diez años para trabajar en las principales universidades e institutos de investigación chinos.

Ciertamente mucho más esfuerzo se ha puesto en Ciencias Técnicas, Ingenierías y Medicina o Economía, pero todavía hay una fuerte necesidad para desarrollar el campo de Humanidades y Ciencias Sociales. Bajo el marco de la nueva llegada de expertos extranjeros a China y los intentos del Ministerio de Educación para atraer a más de ellos, la pregunta es: ¡cuál es el verdadero esfuerzo de las universidades chinas e instituciones académicas para atraer a este grupo de académicos? ¿Existe una plataforma real y un programa de cooperación entre universidades chinas e instituciones académicas europeas? Por razones históricas, estudios contemporáneos sobre China han levantado más interés entre los estudiosos estadounidenses que en los de otros países. Si se consultan los datos aportados (véase el cuadro 1) sobre el porcentaje de profesorado extranjero en universidades chinas que trabajan y residen de forma permanente en tales instituciones se constatará que hay todavía un largo camino por recorrer dado que las cifras son escandalosamente marginales.

Al observar la remuneración mensual para el personal docente e investigador en China, se puede notar que tal baja salarial es un obstáculo notable para captar a investigadores extranjeros por parte de universidades chinas, y por tanto, promover la movilidad internacional en el país (véase el cuadro 1 correspondiente al porcentaje de profesores extranjeros que trabaja en China). El sa- 
Cuadro 1. Porcentaje de académicos extranjeros establecidos de forma permanente en las principales universidades de China

\begin{tabular}{lcc}
\hline & $\begin{array}{c}\text { Total de profesores } \\
\text { en facultades }\end{array}$ & $\begin{array}{c}\text { Profesores extranieros de } \\
\text { forma permanente (\%) }\end{array}$ \\
\hline Universidad de Pekín & 2900 & 0.50 \\
Universidad de Tsinghua & 2941 & 1.60 \\
Universidad de Zhejiang & 2850 & 0.28 \\
\hline
\end{tabular}

Fuente: elaboración propia; http://english.pku.edu.cn/Schools_Departments/; http://www.tsinghua.edu. cn/publish/then/5976/index.html; http://www.zju.edu.cn/english/redir.php? catalog_id=211118

lario oficial nacional dado a un profesor a tiempo completo en China, actualmente establecido por el Ministerio de Educación, ronda alrededor de 4000 RMB (renminbi), siendo aproximadamente 500 euros al mes. Sin embargo, durante los últimos años el gobierno chino ha permitido que departamentos y facultades complementen los salarios oficiales con fondos privados que dichos centros elevan a través de cuotas, consultorías y filiales comerciales.

No obstante, el debate principal no es agrupar académicos y ver quiénes están a favor o en contra sobre la internacionalización de la educación superior en China y quiénes están dispuestos a trabajar permanentemente en aquel país. La cuestión es mucho más profunda, ya que tiene sus raíces en el rígido sistema académico, administrativo y burocrático de las instituciones de investigación y universidades. Se necesitaría una reforma estructural profunda si en realidad los académicos de China quisiesen implementar un currículum sólido relativo a estudios globales, que a su vez incluyera aspectos esenciales e inherentes a este tipo de investigación: movilidad, internacionalización, diversidad y creación de redes académicas. El concepto de estudios globales implica una carga metodológica y teórica vinculada a la interdisciplinariedad de diversas áreas del conocimiento y reconociendo las peculiaridades y características de cada espacio geográfico. La problemática radica en superar, algo que ocurre en la mayoría de los casos, declaraciones no científicas que se manifiestan en forma de sentimientos nacionales y patrióticos, estando muy presentes en el debate académico chino. De este modo, encontramos manifestaciones como: "tanto la globalización y estudios globales son tesis occidentales, carentes de neutralidad..." (Wu Xiaoqun, 2005: 23), u otras como “...no podemos acep- 
tar tal concepto historiográfico, ya que contiene la 'trampa ideológica' de la globalización, es decir, el fin de la soberanía nacional, deterritorialización y desnacionalización de estados-nación, etcétera. Estos conceptos son producto del neoliberalismo de Occidente" (Yu Pei, 2006: 23-24).

Pensar globalmente y sin miopías patrióticas y/o nacionalistas podría abrir nuevas vías de diálogo para fomentar nuevos programas de estudios globales. Sin duda, la práctica de tales planes de estudio en el campo de las Ciencias Sociales y Humanidades conllevaría a algunos problemas metodológicos y limitaciones ideológicas. Una nueva pedagogía debe ser implementada con el fin de establecer nuevos cursos sobre estudios globales, y por tanto, se erradicarían frecuentes obstáculos: como superar las limitaciones burocráticas y administrativas para poner en práctica un programa de estudios globales y, en consecuencia, promover la diversidad y movilidad académica en el personal facultativo.

Con el fin de arrojar luz a esta pregunta es muy importante destacar el poder de "malear" y "moldear" la historia que ha estado muy presente en naciones como China durante época moderna hasta hoy. Civilizaciones, naciones, culturas y continentes todos son construcciones de la llamada fase de la modernidad. Este hecho está muy arraigado en el actual sistema académico chino, a pesar de que algunos investigadores han sido seducidos de alguna manera por la moda actual de emprender estudios globales, principalmente aquellos relacionados con el mundo académico estadounidense con el fin de promover movilidad académica desde China a los Estados Unidos de América. Sin embargo, esto es sólo una forma que ha permitido a la clase dirigente política de China reforzar el pasado e historia nacional del país.

De este modo, los estudios globales se han convertido en China en una nueva narrativa utilizada mediante la reactivación de un modelo neo-confucionista (Elman et al., 2002) cuyo fin es modelar la historia nacional china (Holcombe, 2001: 10), dejando de lado la auténtica finalidad de la práctica y el método del campo de estudios globales que versa en el análisis de la complejidad de las conexiones y relaciones internacionales entre Occidente y Oriente. Como bien dice Dirlik (2005: 391-410), la tarea esencial debe ser analizar el sistema y la organización que articula las relaciones internacionales a escala global alrededor de espacialidades. Tal análisis debe sustituir a la utilización de la historia global como una forma de imaginar el pasado que en última instancia sirve para fomentar y revivir el mito de arcaicas historias nacionales (Anderson, 1983). El objetivo debe ser deconstruir dichas historias nacionales en lugar de construir un nuevo e "imaginado" tiempo nacional. Esta tarea debería ser una "responsabilidad moral" (Bentley, 2005: 51-82). 
Las prácticas departamentales de la historia global han estado mucho más enfocadas en la construcción de historias nacionales y de civilización ancestral de China. En concreto, se pueden hallar dichas prácticas como las relativas al programa "internacional" basado en la antigua ruta de seda china y la actual política y estrategia del gobierno denominada "La Nueva Ruta de Seda: Una Franja, Una Ruta", que podemos encontrar en las facultades de Ciencias Sociales y Humanidades de las universidades chinas.

En este caso, la práctica de la historia, en concreto, y de los estudios globales, en particular, debe implicar un enfoque transnacional mediante la búsqueda de interconexiones y comunicaciones entre China y Occidente. La historia global no se refiere a totalidades estructurales abstractas, sino al enfoque analítico que trata de deconstruir e historizar espacios que convencionalmente han sido ubicados en categorías y disciplinas históricas separadas como puede ser la historia social, política, económica, religiosa, etcétera. La conceptualización de la disciplina de historia global va desde un enfoque "transnacional" a uno "translocal". Esta disciplina describe y analiza, en consecuencia, movimientos creados en estos espacios para tener una comprensión completa de la circulación de personas (diásporas, migraciones), bienes de consumo (a través del comercio de larga distancia) y conocimiento (recepción en diferentes formas de nuevos tipos lingüísticos mediante textos, libros, manuscritos, caligrafía).

Los desafíos inherentes a una completa reforma del sistema de educación superior en China, con base en estudios globales para promover la movilidad, diversidad e internacionalización de las facultades, en la mayoría de los casos están relacionados con fuertes connotaciones socio-culturales y económicas exclusivas a una agenda política muy bien diseñada por parte del gobierno y el Ministerio de Educación; por lo tanto, surge la cuestión de ser chino y/o cuestionar la realidad de China. El paradigma histórico predominante ha identificado a China con los límites de la "China continental".

La política de "China es una y Taiwán es parte de ella", que también abarca a Hong-Kong y Macao, y la población del Este y Sudeste asiático de origen chino, como Singapur y Myanmar, entre otros, se encuentra fuertemente marcada en la agenda política. Esto puede ser identificado como una nueva construcción histórica que legitima el espacio chino y la expansión en una especie de nuevo "imperialismo" moderno. Todo ello relacionado con la corriente neo-confucionista que involucra todos los aspectos de la vida socio-económica, cultural y política, al articular todo lo que se pueda identificar como "chino" que está ampliamente presente en todas las regiones y provincias reactivando un proceso de construcción nacional (March, 1974). 
China no es vista simplemente como una singular nación, es igualmente vista como una civilización con una tradición de larga duración que difiere en muchos aspectos del mundo occidental (Dawson, 1967). No podemos desdeñar estas cuestiones cuando se habla de posibles reformas del sistema de educación superior en China. Por tanto, la pregunta a formular sería: ¿cómo encaja este engranaje político con la concepción y los parámetros de un plan de estudios basado en estudios globales que promuevan diversidad académica? Una declaración sencilla sería afirmar que los planes de estudio departamentales no están bien definidos por los dirigentes políticos, que sin duda tienen como objetivo y misión continuar en una dirección opuesta a lo que venimos proponiendo. Finalmente, otro cuestionamiento sería si realmente se puede escapar de tales restricciones con el fin de construir un programa de estudios globales.

China como otras grandes naciones está formada por diferentes realidades que se pueden observar en sus provincias. La pretensión de la construcción de una "gran nación", unidad de China y el "sueño chino" o chinese dream, concepto claramente emulando al "sueño americano", se deconstruye (Goodman et al., 1994) a través de las realidades multirregionales con una población de diverso origen lingüístico, cultural, religioso, étnico y social. Un concepto muy importante, en este sentido, es el relativo a las diásporas chinas, el cual deconstruye esta aparente homogeneidad social en grupos sociales más dinámicos que han emigrado durante décadas a los Estados Unidos de América y Europa.

Lo anterior contradice y enfrenta la agenda política con las fuerzas históricas. Para ganar esa batalla, el gobierno recientemente ha establecido una política nacional cuyo fin es traer de vuelta los talentos e intelectuales que emigraron a Occidente, sobre todo a los Estados Unidos de América. La llamada internacionalización de la educación superior china y todo su territorio se basa en el reajuste de un nuevo programa, basado en estudios globales con fuertes "características chinas", es decir, una nueva nacionalización del sector académico. La creación de dicho programa está fundamentalmente arraigada en dicha política. ¿Podemos pensar en un plan de estudios global y/o internacional llevado a cabo por meros especialistas y eruditos en estudios globales? ¿Están los programas de estudios globales en China basados en los conceptos de diversidad y movilidad científica que deben ser inherentes al sistema académico? 


\section{POLÍTICA DE CHINA SOBRE LA “FUGA DE CEREBROS”: TRANSFERENCIA DE CONOCIMIENTO Y COOPERACIÓN CIENTÍFICA ENTRE CHINA, LOS ESTADOS UNIDOS DE AMÉRICA Y LA UE}

Como venimos mencionando, en los últimos años el proceso de globalización e integración de mercados se ha acelerado notablemente, hecho que obviamente tiene un gran impacto en la sociedad y economía china. Según Stiglitz (2006: 4) muchos aspectos de la sociedad están más entrelazados y conectados debido a la circulación transnacional de ideas, conocimiento, desarrollo tecnológico, migraciones, bienes de consumo, así como la nueva concienciación de cuestiones medioambientales. En este marco, la transferencia de conocimientos, movilidad de capital humano y material y la consiguiente internacionalización de instituciones y estructuras sociales, es crucial para un crecimiento económico sostenible y modernización de la sociedad a través del rápido desarrollo de la ciencia y tecnología.

Se ha vinculado tal crecimiento y prosperidad a países desarrollados (Lien et al., 2005: 153), a pesar de que hoy día se puede observar que hay una migración masiva de investigadores altamente cualificados procedentes de Europa, especialmente de países como Alemania, Francia o Reino Unido, hecho que está provocando el grave problema de "fuga de cerebros". Esta problemática también acontece en China, sobre todo a partir de la migración masiva de estudiantes y académicos hacia los Estados Unidos de América.

La "fuga de cerebros", por tanto, se puede definir como un problema global en el que los investigadores tienen que emigrar de sus países de origen debido a varias razones: limitado acceso al mercado de trabajo, escasa infraestructura y logística para abrir centros de investigación, así como recortes de financiación a la investigación. En el caso de países en desarrollo, como China, el problema radica en dos factores: el acceso al mercado de trabajo y la insuficiencia en proporcionar los adecuados medios y logística para llevar a cabo proyectos de investigación. En general, la "fuga de cerebros" en países desarrollados y en vías de desarrollo tiene como consecuencia la pérdida permanente de jóvenes talentos y la dificultad en cimentar y desarrollar un sólido sistema de educación superior basado en estándares académicos de alto impacto.

En los últimos seis años, este problema está siendo abordado por China y Europa a través del compromiso en desarrollar una alianza estratégica cuyo fin es promover la movilidad académica en la que tanto las instituciones de origen y acogida se benefician de dicha movilidad. China y Europa han animado a sus estudiantes y académicos a salir al extranjero con la esperanza de que después de un cierto tiempo, tras haber adquirido experiencia en universidades 
internacionales, vuelvan a sus países de origen para transferir el conocimiento adquirido.

A continuación se mostrará brevemente la tendencia histórica y las causas que explican por qué estudiantes y académicos chinos decidieron emigrar a los Estados Unidos de América durante décadas. Esta tendencia se puede observar en la Ley de Protección del Estudiante Chino refrendada en 1992 por el Congreso Norteamericano que establece que estudiantes y académicos chinos puedan quedarse a trabajar e incluso obtener la condición de residente permanente (Cao, 2008: 333). Desde entonces, la principal preocupación del Ministerio de Educación ha sido la "reserva de fuga de cerebros" y "la construcción del país mediante ciencia y educación” (Nawab et al., 2011: 73). En consecuencia, los tres programas más importantes desarrollados por el Ministerio de Educación para conseguir una "ganancia de talentos" son: 1) The Hundred Scholar Program (que se inició en 1994 y está relacionado con científicos de alto nivel y diversa formación para realizar investigaciones en China), 2) National Outstanding Youth Fund (establecido en marzo de 1994 con el objetivo de proporcionar fondos para jóvenes investigadores menores de 45 años de edad que desarrollan su labor en institutos de investigación y universidades), y 3) Changjiang Scholar Project (iniciado en agosto de 1998 para atraer, seleccionar y crear un grupo de investigadores a nivel global cuya finalidad es desarrollar investigación avanzada en todos los campos de la educación superior). Contrastando la Ley de Protección del Estudiante Chino de 1992 podemos observar que Europa inició por su parte una política concreta diseñada para retener y atraer talentos e investigadores, ya fueran de origen europeo o chino, mediante los programas del Consejo Europeo de Investigación, como Marie Curie o el recientemente promulgado programa Horizon 2020.

Para el caso de los programas del gobierno chino, antes mencionados, han tenido un escaso éxito en la retención de expertos en China. El número de investigadores chinos en el extranjero bajo el programa The Hundred Scholar Program ha aumentado de 72 en 1998 a 158 en 2005, con un descenso de 190 en 2000 a 75 en 2003. Desde 2004 a la fecha, el Changjiang Scholar Project ha atraído a 223 estudiantes chinos procedentes de países extranjeros para dedicarse a la investigación científica y enseñanza en los colegios y universidades de China. Pero esta cifra se ve disminuida por la continua tendencia al alza de estudiantes chinos e investigadores que desean continuar su carrera en el extranjero. Según la National Bureau of Statistics (NBS), los datos relativos a estudiantes chinos que salen y regresan de un país extranjero (principalmente a los Estados Unidos de América) desde 1978 a 2006 a través de los ratios de estudiantes repatriados que salen tienden a disminuir desde un 50\% en 1996 
hasta un constante 25\% en 2006 (Cao, 2008: 337) (véase gráfica 1). Los datos representan el hecho de que para los talentos chinos de alto nivel, estudiar, trabajar y vivir en el extranjero resulta más atractivo que volver a China.

Atraídos por un estándar alto de educación y adquisición de mejores condiciones de vida, China encabezó, entre otros países como Corea del Sur, México, Polonia y algunos países de Europa del Este, la proporción total de estudiantes extranjeros en los Estados Unidos de América hacia 2009/10 (alrededor de 127628 estudiantes, 18.4\%) y 2010/11 (alrededor de 157558 estudiantes, $21.8 \%$ ). Los investigadores chinos también superan a los de otros países en la cuota total de académicos internacionales en los Estados Unidos de América desde 2000 hasta 2011, con una cuota total promedio de 20\%. Sin embargo, tal constante flujo secular de estudiantes y académicos chinos a los Estados Unidos de América implica un obstáculo importante para el buen desarrollo de la cooperación estratégica en el ámbito tecnológico y académico entre universidades e institutos de investigación chinos y europeos. Mientras que la preferencia de estudiantes salientes e investigadores chinos es hacia universidades estadounidenses, para los europeos la tendencia en la movilidad es hacia universidades dentro del espacio europeo (véase gráfica 2).

Como puede observarse hay una pequeña tendencia a que doctores de la UE salgan fuera de las fronteras de la propia Unión. La inclinación hacia países asiáticos como China sigue siendo marginal y la participación de los Estados Unidos de América no es mucho mayor en este sentido.

Países europeos han atraído en los últimos años a estudiantes y académicos chinos para estudiar e investigar en diversas instituciones. Sin embargo, Europa sigue siendo un destino marginal en comparación con los Estados Unidos de América en lo referente a estudios chinos. Reino Unido, Alemania y Francia son los principales destinos para académicos y estudiantes de origen chino. Según una investigación apoyada por la Comisión Europea en 2006, la nacionalidad más frecuente de los estudiantes extranjeros en la región EURODATA es la china, aumentando a más del 6\%. Para los años 2002/2003 los estudiantes chinos representan el $12 \%$ del total de los estudiantes extranjeros en el Reino Unido, $8.4 \%$ en Alemania (número 2 después de turcos), y el 4.8\% en Francia (número 3 después de marroquíes y argelinos). ${ }^{6}$ Estos números

Estadísticas de varios años disponibles en la website del Institute of International Education (IIE) <http://www.iie.org/Research-and-Publications/Open-Doors/Data/International-Students/ Leading-Places-of-Origin>

6 EURODATA utiliza 32 países europeos y no europeos, incluyendo a Turquía como origen de instituciones de educación superior (Kelo et al., 2006). 
Gráfica 1. Movilidad de estudiantes chinos (1996-2010)

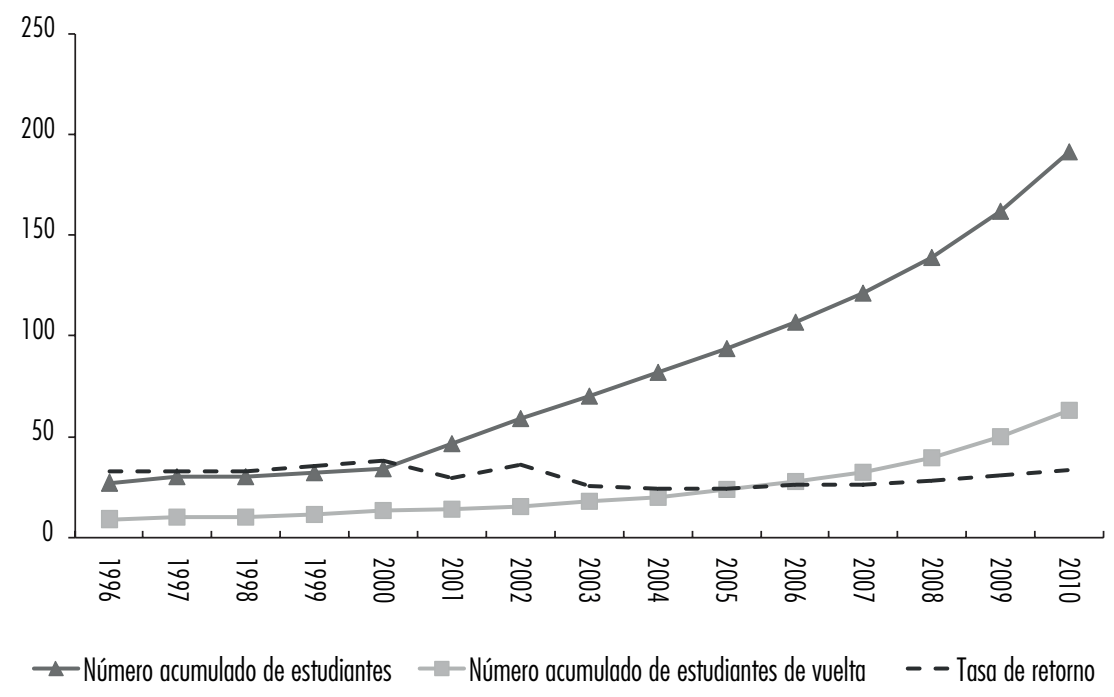

Fuente: elaboración propia. China Statistics Yearbook 1996-2010; los datos de 2008 se basan en datos publicados por el Ministerio de Educación.

son marginales si comparamos las proporciones de estudiantes y académicos chinos que salen hacia los Estados Unidos de América.

Tal laguna en el porcentaje de estudiantes y académicos chinos que van a Europa y europeos que van a China es una de las prioridades a erradicar por parte de la Comisión Europea, hecho que se refleja cuando el Euraxess-Links se estableció en Beijing en 2008, como resultado de las iniciativas emprendidas por la Comisión Europea. Por tanto, el panorama general del sistema de educación superior de China, señalado anteriormente, debe tenerse muy presente en el momento en que los socios europeos tratan de acercarse a las instituciones científicas chinas.

China y Europa en su asociación estratégica de cooperación y movilidad científica han diseñado un nuevo modelo para superar la pesadilla de "fuga de cerebros". Este modelo se basa en la archi-conocida solución del win-win cuya finalidad es transformar la "fuga de cerebros" en "ganancia de talentos" mediante el intercambio de estudiantes y académicos en el extranjero dentro de las acciones Marie Curie y el recientemente lanzado programa Horizon 2020 por la Comisión Europea, así como los nuevos programas del Chinese Scholarship Council en cooperación con la Unesco y el Programa Hanban del Instituto Confucio por el Ministerio de Educación. 
Gráfica 2. Porcentaje de ciudadanos nacionales de la UE con un doctorado habiendo vivido/permanecido en el extranjero en los últimos diez años

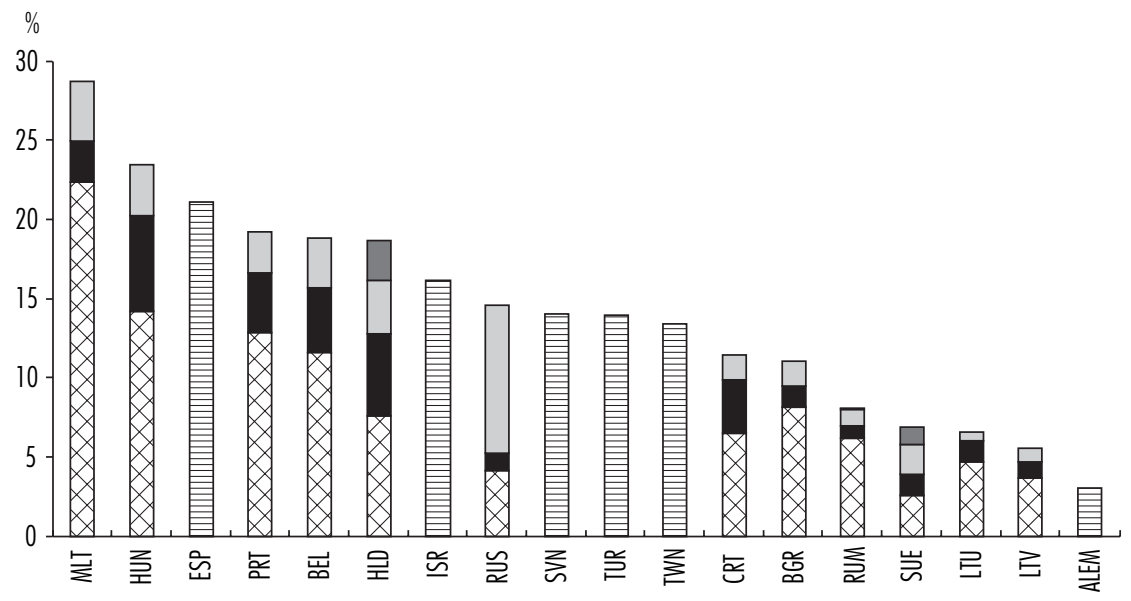

『 Eu27 - Estados Unidos $\square$ Otras economías o desconocido $\square$ Falta de datos $\quad \square$ Desglose no disponible

Notas: MLT (Malta); HUN (Hungría); ESP (España); PRT (Portugal); BEL (Bélgica); HLD (Holanda); ISR (Israel); RUS (Federación rusa); SVN (Eslovenia); TUR (Turquía); TWN (Taiwán); CRT (Croacia); BGR (Bulgaria); RUM (Rumanía); SUE (Suecia); LTU (Lituania); ITV (Latvia); ALEM (Alemania);

"Otras Economías" refiere a aquellas localizadas en África, América, Asia, Europa y Oceanía;

Para Bélgica, Alemania, Países Bajos y España, los datos se refieren solamente a los graduados desde 1990 en adelante. Para Alemania, los datos aportados son relativos a un periodo pequeño de estancia en el extranjero que sería alrededor de seis meses, en comparación a tres meses relativos a otras economías. Para los Países Bajos, Portugal y Rumanía el vacío en la presentación de informes es causado por redondeo en las cifras. Para la Federación rusa, los datos se refieren únicamente a los doctores que trabajan como investigadores y docentes. Para España, la muestra tiene cobertura limitada de doctores para los años desde 2007 a 2009. Para Suecia, el vacío en la información se debe a los datos que no han sido mostrados relativos a ciudadanos nacionales a nivel de cada país y los encuestados que no han sido asignados a países o clasificados como desconocidos. Fuente: OECD Science, Technology and Industry Scoreboard 2011. OECD, basado en OECD/UNESCO Institute for Statistics/Eurostat data collection on careers of doctorate holders 2010, June 2011. 
Dentro de este modelo, China y Europa a través de diversas acciones, invitan a estudiantes y académicos extranjeros a ir a China y Europa con el fin de obtener beneficios a través de sus conocimientos. El Ministerio de Educación chino ha estado llevando a cabo en los últimos años un diálogo con la Comisión Europea en el campo de educación, formación y multilingüismo cultural. Por ejemplo, desde 2004 se seleccionaron más de 2008 estudiantes chinos y 320 académicos para participar en programas conjuntos, cursos y doctorados en virtud del programa Erasmus Mundus. El programa denominado Sciences and Technology Fellowship ha apoyado el intercambio entre estudiantes y académicos europeos y chinos. ${ }^{7}$ En cuanto a la movilidad de la UE a China en 2009, había más de 22600 estudiantes de la UE, principalmente de Reino Unido, Alemania y Francia. La ratio de salientes y entrantes (número de estudiante chino entrante por cada estudiante europeo saliente a China) es de 1:5 para la UE en su conjunto. Esto significa, por cada estudiante europeo que va a China, China exporta cinco estudiantes chinos a Europa. El informe conjunto del estudio realizado entre la Comisión Europea y el Ministerio de Educación de China también da a conocer un dato alarmante sobre el creciente número de estudiantes chinos que no regresan al país después de la graduación.

El esfuerzo conjunto entre Europa y China durante los últimos años ha permitido emprender progresivamente cierto proceso de internacionalización en universidades chinas para contratar investigadores de la UE. Esto supone una gran oportunidad para realizar estudios pioneros por parte de expertos extranjeros que trabajan en las principales instituciones de investigación y, al mismo tiempo, conocer de primera mano el sistema académico de las universidades chinas. Esto constituye el valor añadido más sobresaliente por el mero hecho de trabajar dentro del sistema de educación superior chino. El número de expertos extranjeros trabajando a tiempo completo como personal docente e investigador en departamentos y facultades de universidades chinas es marginal. Por tanto, desde la perspectiva occidental, este hecho explica que todavía haya muchas percepciones erróneas sobre el funcionamiento del sistema académico en China.

Este es un tema verdaderamente inexplorado debido a que la mayoría de expertos occidentales, que trabajan en y sobre China, permanecen durante un corto periodo bajo el estatus de profesor visitante, conferencista o llevan

EU-China Student and Academic Staff Mobility: Present Situation and Future Development, April 2011. Estudio conjunto entre la Comisión Europea y el Ministerio de Educación de China. Disponible en <http://ec.europa.eu/education/external-relation programmes/doc/china/ mobility_en.pdf> 
a cabo su investigación individual patrocinada por una institución europea o americana. Este es un hecho que seguramente empańa y oscurece la percepción académica en Occidente sobre cómo el sistema académico chino funciona y opera en realidad.

Sin embargo, los que permanecen durante un largo tiempo bajo condiciones contractuales chinas trabajando como personal docente e investigador tienen una mejor percepción de cómo el sistema universitario chino funciona en el día a día. En este caso, trabajar en una universidad china constituye un verdadero desafío para investigadores europeos, ya que tienen que enfrentarse a las barreras del idioma y obstáculos de un sistema altamente burocratizado, o lo que vendría a definirse un sistema académico con "características chinas". En la mayoría de los casos el idioma no es el mayor problema, sino el mutuo entendimiento, el cual debe basarse en la comprensión cultural y experiencia internacional de cómo operan instituciones que promueven investigación académica de alta calidad, dejando de lado tintes políticos e ideológicos.

Otro de los desafíos sería confrontar un marcado sistema burocrático, e igualmente solventar el problema de financiación para poder desarrollar proyectos de investigación a través de diferentes programas nacionales. Estas son algunas de las principales dificultades a las que se debe de enfrentar el personal docente y de investigación que trabaja en universidades chinas de forma permanente, pero al mismo tiempo constituyen la parte más fascinante de trabajar ahí. Un mayor entendimiento de tales limitaciones, y búsqueda de soluciones a las mismas, podría permitir una mayor compresión del sistema de educación superior chino.

La solución que de entrada siempre sobresale, aunque requiere un gran trabajo y sacrificio, es la creación de una red científica internacional, formada por expertos académicos occidentales y orientales, que promueva una investigación de calidad en la que obras pioneras puedan llevarse a cabo. Tales acciones vendrían a llenar el gran vacío existente relativo al conocimiento de las Ciencias Sociales y Humanidades del mundo académico chino y europeo. En este sentido debemos citar como ejemplo la creación de la Global History Network en China (GHN) www.globalhistorynetwork.com -que el autor de este artículo fundó y dirige desde el año 2011 al trabajar en la Universidad de Tsinghua, junto con colegas de universidades asiáticas, europeas y americanas-.

La creación de una plataforma académica de esta magnitud tiene como objetivo mantener acuerdos permanentes de cooperación científica entre Europa, América y China, una transferencia dinámica de conocimiento, y fomentar el uso de métodos científicos, técnicas y fuentes de conocimiento 
europeos, americanos y chinos. Además, esto permitirá generar una base que fomente el entendimiento común de diversos sistemas académicos con el fin de que en el largo plazo se pueda alcanzar un reconocimiento mutuo de diplomas de licenciatura, master y doctorado.

Para alcanzar tales objetivos se ha implementado en los últimos años una red científica sino-europea como es la plataforma científica creada por la Comisión Europea (Euraxess-Links), cuya finalidad es contribuir a un mejor conocimiento de las instituciones académicas y establecer proyectos de investigación que den resultados y se puedan aplicar para el desarrollo y fomento de las economías nacionales (véanse gráficas 3, 4 y 5, en las que se pueden apreciar progresivamente el número de expertos europeos de diferentes áreas de investigación y niveles de ocupación que trabajan en China).

Tal plan estratégico se basa en la apertura de nuevos métodos de trabajo y diálogos que fomenten la cooperación entre ambos territorios. La plataforma científica y logística proporcionada por las principales universidades chinas, como la Universidad de Tsinghua, Universidad de Pekín, Universidad de Renmin, Universidad de Fudan y Universidad de Zhejiang, entre otras, va de algún modo en ese sentido, aunque todavía hay un gran trecho por recorrer.

Los recientes acuerdos de cooperación que se firmaron entre instituciones académicas chinas y europeas permiten consolidar permanentemente dicha red científica. Esta cooperación debe basarse en la comprensión del actual sistema universitario que marcha hacia un espacio global basado en la movilidad, diversidad e internacionalización de las estructuras académicas. El reconocimiento de este tipo de conceptos que engloba el mundo académico actual podría permitir una transferencia más fluida de conocimientos científicos, así como el desarrollo del sistema de educación superior. Dicho reconocimiento podría contribuir a mejorar el sistema tanto de universidades europeas como chinas en una amplia escala global para conseguir que instituciones de investigación y universidades sean más competitivas.

El impacto inmediato de proyectos científicos y académicos que promueven movilidad académica, internacionalización del personal que trabaja en universidades e instituciones de investigación y renovación de planes de estudio obsoletos, se refleja en el logro de la fórmula basada en: innovación, calidad en la investigación y el desarrollo científico. Una verdadera y auténtica "ganancia de talentos" puede lograrse a través de la implementación de este modelo. Tal ecuación puede contribuir a un desarrollo económico sostenible mediante el cual todas las estructuras de la sociedad pueden obtener un beneficio en el corto y en el medio plazo. En consecuencia, los problemas relacionados con el medio ambiente, crecimiento demográfico, distribución 


\section{Manuel Pérez García}

Gráfica 3. Nacionalidad de investigadores registrados en el encuentro anual de la Delegación Europea en China (2011)

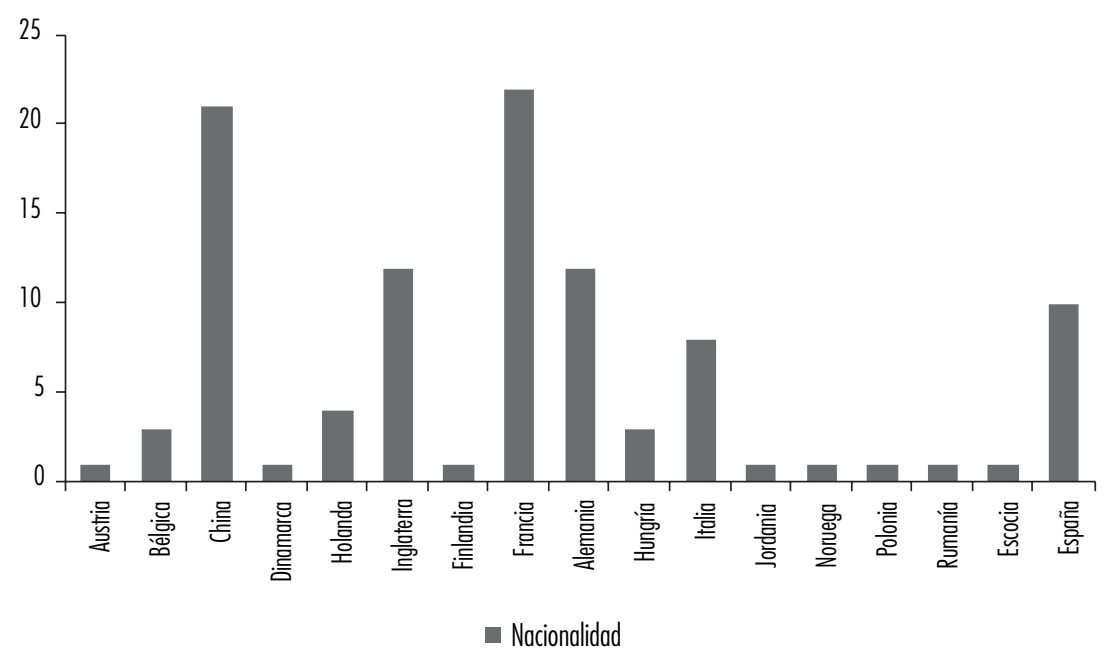

Fuente: elaboración propia. Euraxess in China. China Links Meeting - Mobility of European Researchers to China - Beiiing - 7 December, 2011: Registration list.

Gráfica 4. Área de estudios de investigación

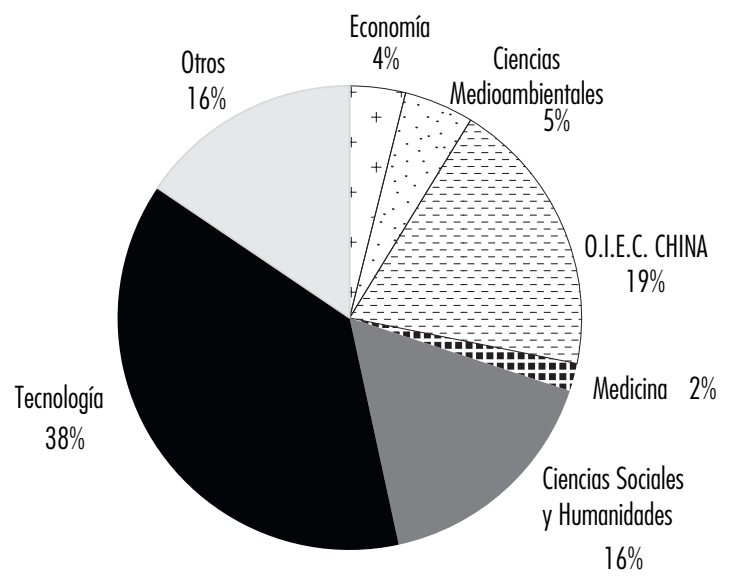

Donde 0.I.E.C. es Organización Internacional de Educación y Ciencia en China.

Fuente: elaboración propia. Euraxess in China. China Links Meeting - Mobility of European Researchers to China - Beiijng 7 December, 2011: Registration List. 
Gráica 5. Ocupaciones

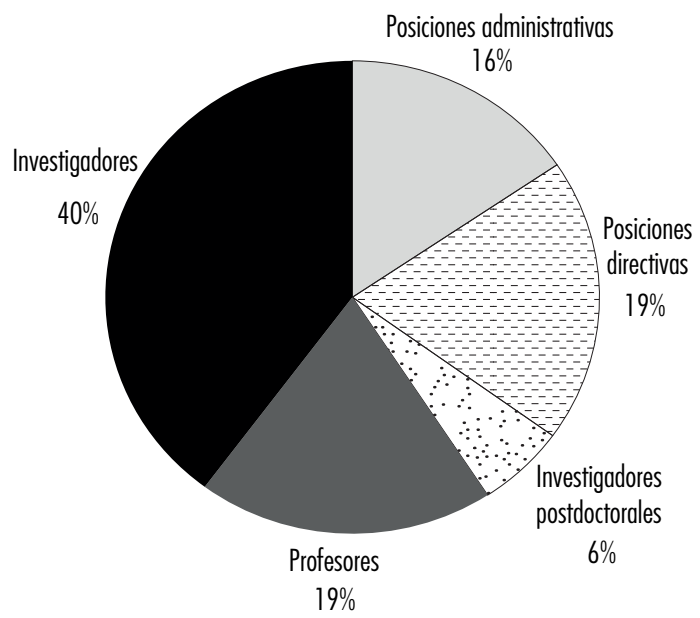

Fuente: elaboración propia. Euraxess in China. China Links Meeting - Mobility of European Researchers to China - Beiijng 7 December, 2011: Registration List.

de riqueza, generación de empleo y desarrollo urbano, entre otras preocupaciones globales, pueden solucionarse de forma más eficaz.

Proyectos piloto de investigación implementados entre China y Europa se basan en la resolución de tales cuestiones. En la gráfica 4 se observa que la proporción de investigadores europeos que van a China es mayor para el área de Ciencias Técnicas, debido a la gran preocupación en resolver problemas de sostenibilidad de zonas urbanas y rurales. Los principales objetivos de universidades chinas y europeas se podrían dividir en los siguientes aspectos:

1. Crear plataformas activas para el intercambio de profesores visitantes entre instituciones europeas y chinas, fomentando el desarrollo de actividades académicas y mejorando la enseñanza y actividades de investigación en disciplinas de interés mutuo. En otras palabras, organizar conjuntamente e implementar ciclos de conferencias, talleres y seminarios que tengan un impacto en la sociedad para la solución de problemas actuales.

2. Intercambio de información científica, académica y técnica, así como otro tipo de información de interés mutuo, por el cual las universidades conserven los derechos de autor y se eviten problemas de plagio y falsificación de datos.

3. Cooperación y asociación de instituciones, con proyectos de investigación en común, en las que tanto investigadores, a nivel individual, como 
equipos de investigación puedan conjuntamente participar con el fin de fomentar proyectos de investigación ambiciosos con un amplio alcance internacional.

Dentro de este marco de colaboración y cooperación mutua, las instituciones chinas y europeas se están beneficiando de la excelencia y competitividad que promueve el intercambio académico a escala global e internacional. La comunicación permanente entre instituciones puede facilitar la transferencia de información de alto valor, por la cual se pueden alcanzar los objetivos de investigación establecidos, así como ciertas mejoras en los programas de intercambio de estudiantes y profesorado. Una de las principales lagunas que existen en algunas instituciones europeas, sobre todo las relativas a países mediterráneos de la UE, es su bajo nivel de internacionalización y conocimiento sobre el funcionamiento de otros grupos de investigación internacionales.

En relación con algunos objetivos marcados a través de actividades de divulgación se debe tener como principio fundamental la creación de una conciencia profunda y adecuada comprensión social de la importancia de un nuevo modelo global de movilidad académica y educación superior. Bajo estos parámetros, es importante difundir y concienciar a la sociedad sobre la importancia y aplicación de actividades de investigación, elemento que debe ser comprensible y tangible para los no especialistas en la materia.

En consecuencia, hay que mejorar y fomentar lo que podría definirse como "concienciación pública" de la ciencia e investigación de vanguardia. Debe haber un contacto muy directo con el público en general con el fin de ayudar a los investigadores a comprender plenamente las necesidades públicas y promover el interés por la ciencia y tecnología e igualmente emprender actividades de divulgación que hagan adquirir a la sociedad tal "conciencia" de la labor emprendida por investigadores y académicos.

Así pues, a través de una concreta pedagogía y un comprensible método de difusión del conocimiento científico e investigación, sería más fácil desarrollar un aprendizaje público de tal "concienciación científica". Tal tipo de actividades de difusión tienen como principal objetivo difundir en la sociedad la importancia del ámbito de las Ciencias Sociales, y cómo la investigación interdisciplinaria puede ayudar a entender las dinámicas e interconexiones de una sociedad globalizada, que en nuestro caso abarca campos tan dispares, pero igualmente interconectados como son las Relaciones Internacionales, Historia, Economía o Sociología. 


\section{CONCLUSIONES}

En el momento de aproximarnos al sistema de educación superior chino es necesario tener en cuenta el grado de internacionalización de las estructuras académicas y científicas de las distintas universidades e institutos de investigación.

Todavía hay un largo camino por recorrer, ya que hay muchos obstáculos que impiden una cooperación fluida entre universidades y centros de investigación chinos y occidentales debido a las limitaciones institucionales que impiden implementar un modelo global basado en la diversidad, movilidad e internacionalización de las instituciones académicas. Igualmente tenemos que señalar que hay un bajo nivel de conocimiento en Europa sobre el sistema de educación superior chino, siendo un tema que se explica principalmente por el número marginal de profesores e investigadores europeos (igualmente esto se aplicaría para el caso americano) que trabajan en China de forma permanente. Los acuerdos de cooperación científica hasta la fecha, especialmente la multiplicación de memorándums de entendimiento, son de escaso y limitado alcance e igualmente insuficientes para establecer un marco general y concreto de cooperación a nivel institucional. Estos acuerdos serían más operativos si se fundamentasen a nivel departamental o individual, de investigador a investigador, cuando realmente se buscan intereses científicos y sinergias comunes.

De esta forma, conociendo de antemano las condiciones y los obstáculos inherentes al propio sistema, sería más comprensible y realista atajar el problema mediante la implementación de sólidas redes científicas que servirían como plataforma permanente para un común entendimiento sobre la base de confianza mutua y transparencia. Estos son los ingredientes para lograr el archi-conocido win-win entre socios y grupos científicos. Bajo este marco se podría lograr una reforma real de los planes de estudio y programas científicos basados en estudios globales mediante la promoción de diversidad, movilidad e internacionalización. En este sentido, las dificultades señaladas anteriormente, inherentes a diferentes modelos de educación superior que operan en Europa y China, y que caracterizan a un sistema altamente burocrático y falto de meritocracia, se verían progresivamente erradicadas. Por tanto, es necesario encontrar sinergias entre instituciones de investigación con el fin de hallar intereses comunes de cooperación e invertir todos los esfuerzos en proyectos de investigación muy concretos para así comparar y comprender los divergentes o convergentes procesos socio-económicos de China, América, Europa, incluso África, en lugar de perpetuar la confrontación de larga duración entre los poderes hegemónicos de Occidente y Oriente. 


\section{BIBLIOGRAFÍA}

Anderson, Benedict (1983), Imagined Communities: Reflections on the Origin and Spread of Nationalism, London, Verso.

Bentley, Jerry (2005), "Myths, Wagers, and Some Moral Implications of World History", Journal of World History, 16, pp. 51-82.

Cao, Cong (November 2008), "China’s Brain Drain at the High End: Why Government Policies gave failed to Attract First-rate Academics to Return", Asian Population Studies, vol. 4, n. 3.

Dawson, Raymond (1967), The Chinese Chameleon: An Analysis of European Conceptions of Chinese Civilization, London, Oxford University Press.

Dirlik, Arif (December 2005), "Performing the World: Reality and Representation in the Making of World Histor (ies)", Journal of World History, vol. 16, n. 4, pp. 391-410.

Elman, Benjamin, Duncan, John and Ooms, Herman (eds.) (2002), Rethinking Confucianism: Past and Present in China, Japan, Korea, and Vietnam, Los Angeles, ucla Asian Pacific Monograph Series.

EU-China Student and Academic Staff Mobility: Present Situation and Future Development (April 2011), Estudio conjunto entre la Comisión Europea y el Ministerio de Educación de China. Disponible en <http://ec.europa. eu/education/external-relation programmes/doc/china/mobility_en.pdf>

Fairbank, John and Kwang-Ching Liu (eds.) (1950), Modern China. A Bibliographical Guide to Chinese Works, 1898-1937, Cambridge, Harvard University Press.

Geeraerts, Gustaaf (2011), "China, the EU, and the New Multipolarity", European Review, vol. 19, n. 1, pp. 57-67.

Goodman, David S.G. and Segal, Gerald (1994), China Deconstructs: Politics, Trade and Regionalism, London, Routledge.

Hardin, Harry (1993), "The Evolution of American Scholarship on Contemporary China”, en Shambaugh, David (ed.), American Studies on China Contemporary China, Washington, Woodrow Wilson Center Press, pp. 14-42.

Holcombe, Charles (2001), The Genesis of East Asia: 221 B.C. - A.D. 907, Hawaii, University of Hawaii Press.

Hu-Dehart, Evelyn (1998), "The Chinese Diaspora”, in Leonard, G. (ed.), The Asian Pacific American Heritage, Garland Press.

Institute of International Education (IIE) <http://www.iie.org/Research-andPublications/Open-Doors/Data/International-Students/Leading-Placesof-Origin> 
Kelo, Maria, Teichler, Ulrich and Wächter, Bernd (eds.) (2006), EURODATA: Student Mobility in European Higher Education [pdf] Available at: <http:// ec.europa.eu/education/erasmus/doc/publ/eurodata_en.pdf>

Lien, Donald and Wang, Yang (March 2005), "Brain Drain or Brain Gain: A Revisit", Journal of Population Economics, vol. 18, n. 1.

Lindbeck, John (ed.) (1971), China: Management of Revolutionary Society, Seattle, University of Washington Press.

Mackerras, Colin (2000), Western Images of China, Oxford, Oxford University Press, 2 edition.

March, Andrew (1974), The Idea of China: Myth and Theory in Geographic Thought, New York, Praeger.

Morse, Hosea B. (1910-1918), The International Relations of the Chinese Empire, London, New York, Longmans, Green, and Co., 3 v.

(1921), The Trade and Administration of China, $3^{\text {rd }}$ edition, Shanghai, Kelly and Walsh.

Nawab, Samina and Shafi, Khuram (2011), "Retaining the Brains, Policies Adopted by P.R.China to Attract and Retain Research Talent", Australian Journal of Business and Management Research, vol. 1, n. 4, pp. 72-77.

Remer, Carl (1928), The Foreign Trade of China, Shanghai, The Commercial Press Limited.

Said, Edward (1978), Orientalism, London, Penguin.

Schell, Orville (1977), In the People's Republic: An American's Firsthand View of Living and Working in China, Random House.

Shambaugh, David (ed.) (1993), American Studies on China Contemporary China, Washington, Woodrow Wilson Center Press.

Shen Qiu (2009), "Subtle Changes in Relations among Key Players in the Reform of the International Financial and Economic System", Foreign Affairs Journal, 21(92), pp. 33-55.

Stiglitz, Joseph (2006), Making Globalization Work, New York, W.W. Norton \& Company.

Wu, Xiaoqun (2005), “Do We Really need a 'Global View of History'?”, Xuesbu Yanjiu (Academic Research) 1.

Yu, Pei (2006), "Global History and National Historical Memory", Shixuelilunyanjiu (Historiography Quarterly) 1. 
